\title{
Summary of clinical trials in rheumatoid arthritis using infliximab, an anti-TNF $\alpha$ treatment
}

\author{
Gregory Harriman, Lesley K Harper, Thomas F Schaible
}

Cytokines play a central part in the human immune response and can generally be categorised as proinflammatory or anti-inflammatory cytokines in nature. Tumour necrosis factor $\alpha$ $(\mathrm{TNF} \alpha)$ is a proinflammatory cytokine and is a key mediator of inflammation. It has been shown to be pivotal early in the inflammatory cascade. Its effect on upregulation of inflammatory events within the joints makes it an appropriate pharmacological target in rheumatoid arthritis (RA). Infliximab, an anti-TNF treatment, has been successful in clinical trials in reducing $\mathrm{TNF} \alpha$ induced production of other inflammatory mediators, thereby reducing the signs and symptoms of RA.

Infliximab is a chimeric monoclonal antibody that is $75 \%$ human and $25 \%$ mouse protein. ${ }^{1}$ The mouse portion contains a variable region binding site while the human portion is responsible for effector function. Infliximab binds to soluble $\mathrm{TNF} \alpha$ as well as membrane bound $T N F \alpha$. It has also been shown to lyse $\mathrm{TNF} \alpha$ producing cells in vitro, although this has not been studied in vivo. ${ }^{2}$ The antibody binds with high affinity, avidity and specificity to human $\mathrm{TNF} \alpha$. It does not bind to $\mathrm{TNF} \beta$, otherwise known as lymphotoxin- $\alpha$. This high specificity decreases the potential for non-specific effects on other biological pathways. There is a dose proportional neutralisation of $\mathrm{TNF} \alpha$ at doses ranging from 0.01 to 10 $\mathrm{mg} / \mathrm{kg}$ in subjects with increased $\mathrm{TNF} \alpha$ levels after endotoxin challenge. Because infliximab is a protein, it is not metabolised by cytochrome P-450 enzymes providing additional advantages. For example, the genetic polymorphisms of P-450 isoenzymes and the consequent variability in metabolism leading to different toxic or inactive metabolites are less of an issue. Also, the likelihood of complex drug

Correspondence to: Dr G Harriman. interactions are decreased in comparison with small molecules.

Infliximab has also been studied in inflammatory bowel disease, specifically Crohn's disease. $\mathrm{TNF} \alpha$ seems to play a central part in the inflammatory response in patients with active Crohn's disease. TNF $\alpha$ levels are increased in bowel mucosa, and after treatment with infliximab, $\mathrm{TNF} \alpha$ levels decreased over the four weeks after treatment. ${ }^{3}$ Unlike current immunosuppressive treatments, such as corticosteroids, infliximab not only provides symptomatic relief but also heals colonic ulcerations. ${ }^{4}$ As table 1 shows, infliximab is highly effective in providing clinical improvement $(>70$ point improvement in the Crohn's Disease Activity Index (CDAI), an index comprising signs and symptoms of the disease), inducing even remission (CDAI < 150) in moderately to severely active patients and also in fistulising Crohn's disease patients who are refractory to conventional treatment. ${ }^{5}{ }^{6}$

There have been several key clinical trials in RA with infliximab. Over 1000 patients have been treated with infliximab in clinical trials. Two open label and four placebo controlled, randomised, double blinded trials in approximately 650 RA patients have been conducted. Of the 650 patients, 555 have been treated with infliximab, including 342 patients in a large phase 3 study (ATTRACT). In ATTRACT, patients were treated for over one year. All trials include long term follow up for three years for all patients after the trial's completion.

Serum measurements of infliximab concentration have been obtained in all patients in clinical trials, providing a comprehensive evaluation of pharmacokinetics. A predictable pharmocokinetic profile has been obtained, where maximal serum concentration $\left(\mathrm{C}_{\max }\right)$ and area under the curve (AUC) is proportional to the dose. $^{7}$ The volume of distribution is consistent with primarily an intravascular distribution. Infliximab has a terminal half life of 8-12 days. No accumulation of infliximab with repeated dosing was observed, indeed dose proportionality was maintained with repeat dosing. ${ }^{7}$ The $3 \mathrm{mg} / \mathrm{kg}$ every eight weeks regimen used in ATTRACT provides serum levels out to eight weeks, which have been shown to neutralise $\mathrm{TNF} \alpha$ activity.

Earlier studies indicated a dose response with infliximab in RA. ${ }^{8}$ As evaluated by both Paulus 20 and Paulus 50 criteria, a single infusion of $1 \mathrm{mg} / \mathrm{kg}$ had activity but provided less response than a single infusion of $10 \mathrm{mg} / \mathrm{kg}$ (see table 2). Within three days, there was a rapid decrease of $\mathrm{C}$ reactive protein (CRP). Notably, $1 \mathrm{mg} / \mathrm{kg}$ was not as effective as $10 \mathrm{mg} / \mathrm{kg}$ in

Ninety four patients were randomly assigned to one of three treatment groups ( $5 \mathrm{mg}$ $\mathrm{mg} / \mathrm{kg}$ infliximab or placebo given at weeks 0,2 and 6$)$. Results are shown for the recommended dose of $5 \mathrm{mg} / \mathrm{kg}$. 
Table 2 Clinical results with a single infusion of infliximab in patients who had failed $D M A R D s$

\begin{tabular}{llll}
\hline & Placebo & 1 mg/kg infliximab & 10 mg/kg infliximab \\
\hline Paulus 20 (\% patients) & 8 & 44 & 79 \\
Paulus 50 (\% patients) & 8 & $\mathrm{p}=0.008$ & $\mathrm{p}<0.0001$ \\
\% reduction in SJC & 28 & $\mathrm{p}=0.138$ & 58 \\
& & 40 & $\mathrm{p}<0.0005$ \\
\% reduction in TJC & 6 & $\mathrm{p}<0.001$ & $\mathrm{p}<0.001$ \\
& & 42 & 60 \\
& & $\mathrm{p}<0.001$ & $\mathrm{p}<0.001$ \\
\hline
\end{tabular}

Adapted from Elliot et al. ${ }^{8}$

Patients had failed treatment with at least one DMARD and did not receive methotrexate during the trial. Patients were withdrawn from their DMARD treatments at least four weeks before entry into the study. The study population totalled 72 patients. Data shown are results at four weeks after a single infusion.

providing a sustained decrease in CRP concentrations.

Another study evaluated the efficacy of infliximab both with and without concomitant methotrexate (MTX) using Paulus 20 criteria. $^{7}$ This was a placebo controlled, randomised, dose ranging trial in patients with RA. Entry criteria included diagnosis with RA by American College of Rheumatology (ACR) criteria and active disease despite treatment with MTX. Patients had to be on MTX for at least six months and had to be on a stable dose of $7.5 \mathrm{mg} /$ week before enrollment. Additionally, concomitant stable doses of corticosteroids and/or non-steroidal anti-inflammatory drugs (NSAIDs) were permitted. Patients were randomised to one of seven treatment groups, $1 \mathrm{mg} / \mathrm{kg}, 3 \mathrm{mg} / \mathrm{kg}$, and $10 \mathrm{mg} / \mathrm{kg}$ of infliximab, with or without $7.5 \mathrm{mg} / \mathrm{week}$ of MTX compared with a placebo infusion and $7.5 \mathrm{mg} /$ week of MTX. Patients were treated with multiple infusions at weeks $0,2,6,10$ and 14. The doses yielding the highest response rates, up to $80 \%$, included $3 \mathrm{mg} / \mathrm{kg}$ and 10 $\mathrm{mg} / \mathrm{kg}$ of infliximab, either with or without MTX (see table 3 ). In patients receiving the 3 $\mathrm{mg} / \mathrm{kg}$ and $10 \mathrm{mg} / \mathrm{kg}$ doses without MTX, responses were observed in the majority of patients treated, although the response rates were slightly less than those in patients receiv-

Table 3 Clinical results in patients receiving multiple infusions of infliximab with without MTX

\begin{tabular}{|c|c|c|c|c|c|}
\hline & \multirow{2}{*}{$\frac{\text { Placebo }}{\text { MTX+ }}$} & \multicolumn{2}{|c|}{$3 \mathrm{mg} / \mathrm{kg}$ infiximab } & \multicolumn{2}{|c|}{$10 \mathrm{mg} / \mathrm{kg}$ infiximab } \\
\hline & & $M T X+$ & $M T X^{-}$ & $M T X^{+}$ & $M T X_{-}$ \\
\hline \multicolumn{6}{|c|}{ Paulus 20 (\% patients) } \\
\hline 2 weeks & 7 & 27 & 43 & 62 & 53 \\
\hline 14 weeks & 0 & 60 & 57 & 79 & 53 \\
\hline 26 weeks & 7 & 47 & 21 & 57 & 33 \\
\hline \multicolumn{6}{|c|}{ Paulus 50 (\% patients) } \\
\hline 2 weeks & 0 & 7 & 29 & 15 & 20 \\
\hline 14 weeks & 7 & 60 & 36 & 62 & 40 \\
\hline 26 weeks & 0 & 40 & 14 & 50 & 20 \\
\hline \multicolumn{6}{|c|}{$\%$ improvement in TJC } \\
\hline 2 weeks & 0 & 43 & 55 & 65 & 39 \\
\hline 14 weeks & $\mathrm{N} / \mathrm{A}^{\star}$ & 71 & 81 & 96 & 52 \\
\hline 26 weeks & $\mathrm{N} / \mathrm{A}^{\star}$ & 90 & 61 & 96 & 43 \\
\hline \multicolumn{6}{|c|}{$\%$ improvement in SJC } \\
\hline 2 weeks & 18 & 25 & 47 & 43 & 63 \\
\hline 14 weeks & $\mathrm{N} / \mathrm{A}^{\star}$ & 75 & 76 & 80 & 63 \\
\hline 26 weeks & $\mathrm{N} / \mathrm{A}^{\star}$ & 73 & 29 & 70 & 74 \\
\hline
\end{tabular}

Adapted from Maini et al. ${ }^{7}$

One hundred one patients with an incomplete response or flare of rheumatoid arthritis activity while receiving MTX were studied. Patients receiving concomitant MTX continued on doses of $7.5 \mathrm{mg} /$ week, beginning four weeks before randomisation. Infusions of infliximab were given at weeks $0,2,6,10$ and 14 . Patients were followed up for 26 weeks.

* Not available: The number of patients remaining in the trial was less than $50 \%$ of the number of patients randomised, therefore summary statistics were not determined.

TJC = tender joint count, SJC = swollen joint count. ing the same doses with MTX. The response was not as well sustained once infliximab treatment was stopped at week 14 in patients not receiving concomitant MTX treatment. Three $\mathrm{mg} / \mathrm{kg}$ and $10 \mathrm{mg} / \mathrm{kg}$ of infliximab, both with and without MTX, also produced a high level of clinical response, as assessed by Paulus 50 criteria. In addition, when the ACR2 0 and ACR50 criteria were used, comparable levels of response were observed as for the Paulus 20 and 50 criteria. The $3 \mathrm{mg} / \mathrm{kg}$ and $10 \mathrm{mg} / \mathrm{kg}$ groups also showed substantial, near remission reductions in swollen joint counts (SJC) and tender joint counts (TJC). These levels were also sustained better when infliximab was given in combination with MTX.

ATTRACT is the largest clinical trial to date of an anti-TNF $\alpha$ agent in patients with RA. It is a phase 3, placebo controlled, randomised, double blind trial in 428 patients with active RA despite treatment with MTX. Entry criteria for this trial included diagnosis of RA by ACR criteria, active disease at screening despite treatment with MTX, and patients had to be taking MTX for at least three months at a stable dose of $\geqslant 12.5 \mathrm{mg} /$ week for at least four weeks. All patients continued to receive MTX during the study and were randomised to receive placebo, or $3 \mathrm{mg} / \mathrm{kg}$ or $10 \mathrm{mg} / \mathrm{kg}$, and after receiving infusions at weeks 0,2 and 6 , continued to receive the assigned treatment every four or eight weeks. All treatment groups were well balanced for demographic and baseline disease characteristics. In general, patients had advanced, aggressive RA, and roughly 50\% were in functional class III. Most of these patients had longstanding disease with a median duration of 8.4 years. They had failed a median of three disease modifying antirheumatic drugs (DMARDs), including MTX. Approximately $50 \%$ of the patients had been taking MTX for three or more years, roughly $80 \%$ were rheumatoid factor (RF) positive, and notably, $37 \%$ had prior joint surgery, with one quarter having had prior joint replacements.

The primary end point of ATTRACT was the achievement of ACR 20 response criteria at 30 weeks. Additional end points are to be evaluated at one year ( 54 weeks) after the onset of treatment; notably prevention of structural damage by radiological assessment. At two years of treatment, patients will be evaluated to support a reduction in physical disability. The primary efficacy analysis, based on ACR 20, showed that $50-60 \%$ of patients achieved a clinical response at 30 weeks compared with only $20 \%$ of MTX control patients. This response rate was highly statistically significant $(\mathrm{p}<0.001)$ for each infliximab treated group compared with the MTX control group. In addition, the $3 \mathrm{mg} / \mathrm{kg}$ every eight weeks was as effective as the higher $10 \mathrm{mg} / \mathrm{kg}$ dose or the more frequent four week interval. Given the severity of these patients' disease and their refractory nature to other treatments, particularly MTX, these responses support the unique therapeutic benefit of infliximab treatment.

The ACR 20 responses were rapid and sustained over 30 weeks. No trend for diminishing responsiveness was observed over 30 
weeks, suggesting that antibodies against infliximab, if they occurred, were not manifested clinically. (Although samples for human antichimeric antibodies (HACA) are being collected for all patients, they cannot be evaluated in a valid manner while patients are still receiving treatment, because of the continued presence of infliximab in the serum. Thus complete HACA analysis cannot be finished until the trial is completed.) In evaluating CRP concentrations over time, a rapid normalisation of CRP concentrations was observed. The normalisation was sustained over 30 weeks. The ability to normalise CRP rapidly and to maintain the normal values over time demonstrates the ability of infliximab to profoundly suppress inflammation, which may potentially prevent structural damage. Data evaluating prevention of structural damage will be evaluated and presented at a later date.

In evaluating patients who stopped treatment, it was observed that infliximab was well tolerated; $5 \%$ of infliximab patients dropped out because of adverse events, compared with $8 \%$ in the MTX control group. Drop outs attributable to lack of efficacy were much higher in the MTX control group, $25 \%$, compared with $8 \%$ in the infliximab groups. The high MTX control drop out rate underscores the severity of RA in these patients who were already on MTX.

Some adverse events were reported. The most common of these was upper respiratory tract infection. Other fairly common adverse events included headache, nausea, sinusitis, rash and coughing. It is important to note that these adverse events did not increase over time. The incidence of serious adverse events was comparable between the infliximab treated groups and MTX control group. Infections treated with antimicrobials were similar to MTX control in the $3 \mathrm{mg} / \mathrm{kg}$ every eight weeks dose group, although somewhat higher with higher doses. The incidence of infusion reactions was low. Most infusion reactions were mild and no serious infusion reactions were reported. There was no trend for an increase in the number or severity of infusion reactions over time, again suggesting that any antibodies that may have developed against infliximab did not have significant clinical implications.

In a study conducted in patients with Crohn's disease to assess delayed hypersensitivity events, 10 of 40 patients treated two to four years after their initial therapy experienced delayed infusion related reactions. Their symptoms consisted predominantly of myalgia and/or arthralgia, fever and/or rash occurring 3-12 days after infusion. Nine of these 10 patients received an earlier liquid formulation of infliximab that is no longer in use. Delayed hypersensitivity events have only rarely been observed in patients receiving infliximab retreatment at intervals of one year or less.

The incidence of HACA has been low (13\%) at recommended doses in Crohn's disease patients. Titres are generally low (two thirds had titres $: \leqslant 1: 40$ ) and usually not associated with significant clinical events. The incidence of HACA formation in RA patients is approxi- mately $10 \%$ in infliximab phase 2 clinical trials using comparable dose regimens as used in the ATTRACT trial. In evaluating these data, it should be emphasised that antibody formation occurs with virtually all biological treatments and current debate focuses on the clinical relevance of antibody development. In this regard, it is important to note that there have been HACA positive patients given multiple infusions of infliximab who did not experience clinically diminished efficacy or infusion reactions. As discussed earlier, in the ATTRACT trial, analysis of HACA incidence cannot be done until patients complete treatment. However, the sustained efficacy and low incidence of infusion reactions over time in ATTRACT suggest that clinically significant HACA development is infrequent.

In previous studies with infliximab, approximately $9 \%$ of patients developed anti-doublestranded DNA (dsDNA) antibodies. In ATTRACT, $16 \%$ of patients developed antidsDNA at some time during the 30 weeks, but generally the levels were low and variable in occurrence and rarely lead to clinically significant complications. Drug induced lupus occurred in less than $0.5 \%$ of patients treated with infliximab, all of whom were successfully treated medically, and none of these patients developed renal complications or other major organ damage.

The incidence of malignancies with infliximab treatment in the ATTRACT study was not higher than expected based upon the NIH SEER database of an age and sex matched general population. Three patients in the ATTRACT study, all of whom received infliximab, developed malignancies while two to three patients would be expected to have malignancies based on the NIH SEER database. In all infliximab trials, three RA patients out of 555 infliximab treated patients have developed lymphoma either during trial participation or during three years of follow up. All of these patients had longstanding RA. An increased risk of developing lymphoma in RA is well established and seems to be correlated with the degree of inflammation and/or duration of disease. In addition, one Crohn's patient out of 200 treated patients and one AIDS patient in other studies also developed lymphoma.

Overall, infliximab seems well tolerated in patients with Crohn's disease and RA when given as a single or multiple dose regimen. Potential adverse experiences of infliximab treatment can be monitored and are responsive to medical treatment and to discontinuation of treatment. Patients with RA face a lifetime of potentially debilitating disease. The progressive, chronic course and sequelae of RA require long term treatment to maximise the antiinflammatory effects on the joints and minimise the systemic impact of treatment. Treatment with infliximab in patients with moderate to severe RA rapidly produced a reduction in the signs and symptoms of this disease. The positive results obtained in the ATTRACT trial, a large phase 3 study in RA that is treating patients for two years, have confirmed these findings. The benefit of infliximab has been 
consistent in all six clinical trials (four placebo controlled, randomised, double blind and two open label) conducted with infliximab in this disease. The advent of infliximab holds promise for the effective treatment of active RA that is well tolerated.

The data in this paper were presented at the "Advances in Targeted Therapies: TNF $\alpha$-Blockade in Clinical Practice" seminar held in Montego Bay, Jamaica in March of 1999

1 Knight DM, Trinh H, Le J, et al. Construction and initial characterization of a mouse-human chimeric anti-TNF antibody. Mol Immunol 1993;30:1443-53.

2 Scallon BJ, Moore MA, Trinh H, Knight DM, Ghrayeb J. Chimeric anti-TNF- $\alpha$ antibody cA2 binds recombinan transmembrane $\mathrm{TNF}-\alpha$ and activates immune effector functions. Cytokine 1995;7:251-9.

3 Baert FJ, D'Haens GR, Peeters M, et al. Tumor necrosis factor a antibody (infliximab) therapy profoundly down- regulates the inflammation inCrohn's ileocolitis. Gastroenterology 1999;116:22-8

4 D'haens G, van Deventer S, van Hogezand R, et al. Endoscopic and histological healing with infliximab anti-tumor necrosis factor antibodies in Crohn's disease: a European multicenter trial. Gastroenterology 1999;116: 1029-34

5 Targan SR, Hanauer SB, van Deventer SJ, et al. A short-term study of chimeric monoclonal antibody cA2 to tumor necrosis factor a for Crohn's disease. N Engl J Med 1997;337:1029-35.

6 Present DH, Rutgeerts P, Targan S, et al. Infliximab for the treatment of fistulas inpatients with Crohn's disease. N Engl J Med 1999;340:1398-405.

7 Maini RN, Breedveld FC, Kalden JR, et al. Therapeutic efficacy of multiple intravenous infusions of anti-tumor necrosis factor $\alpha$ monoclonal antibody combined with low-dose weekly methotrexate in rheumatoid arthritis. Arthritis Rheum 1998;41:1552-63.

8 Elliot MJ, Maini RN, Feldmann M, et al. Randomised doubleblind comparison of chimeric monoclonal antibody to tumor necrosis factor $\alpha$ (cA2) versus placebo in rheumatoid arthritis. Lancet 1994;344:1105-10. 